antibiotic cocktail pretreatment at the end of experiment)( IDDF2021-ABS-0196 Figure1B. Clostridium butyricum and its supernatant ameliorated colitis and regulated immune cells; The macroscopic images of colon)( IDDF2021-ABS-0196 Figure 1C. Clostridium butyricum and its supernatant ameliorated colitis and regulated immune cells; The statistical analysis of colon length). The results of qPCR showed that the relative expression of pro-inflammatory cytokines TNF- $\alpha$, IL- 6 , IL- $1 \beta$, IL-17a were down-regulated after CBM and its supernatant treatment. And the relative expressions of anti-inflammatory cytokines TGF- $\beta$, IL-10, IL-4, IL-5, IL-13 were up-regulated (IDDF2021-ABS-0196 Figure1F. Clostridium butyricum and its supernatant ameliorated colitis and regulated immune cells; The amount of Th1 cells based on flow cytometry, and its statistical analysis). Flow cytometry showed that the amount of neutrophils, macrophage, Th1, Th17 cells were down-regulated which were the pro-inflammatory immune cells in intestinal mucosa lamina propria after $\mathrm{CBM}$ and its supernatant treatment (IDDF2021-ABS-0196 Figure1D. Clostridium butyricum and its supernatant ameliorated colitis and regulated immune cells; The amount of neutrophil based on flow cytometry, and its statistical analysis) (IDDF2021-ABS-0196 Figure1E. Clostridium butyricum and its supernatant ameliorated colitis and regulated immune cells; The amount of macrophage based on flow cytometry, and its statistical analysis) (IDDF2021-ABS-0196 Figure1F. Clostridium butyricum and its supernatant ameliorated colitis and regulated immune cells; The amount of Th1 cells based on flow cytometry, and its statistical analysis) (IDDF2021-ABS-0196 Figure1G. Clostridium butyricum and its supernatant ameliorated colitis and regulated immune cells; The amount of Th17 cells based on flow cytometry, and its statistical analysis) (IDDF2021-ABS0196 Figure $1 \mathrm{H}$. Clostridium butyricum and its supernatant ameliorated colitis and regulated immune cells; The relative expression of inflammatory cytokines)

Conclusions CBM can alleviate the symptoms and colon shortening of mice with colitis after pre-treatment with $\mathrm{ABx}$, improve the expression of inflammatory factors, and might play an anti-inflammatory role by down-regulating the infiltration of inflammatory immune cells in intestinal mucosa lamina propria.

\section{IDDF2021-ABS-0197 DELAYED INTERVENTION OF AGAROPECTIN-DERIVED OLIGOSACCHAR- IDES ALLEVIATE LIPID ACCUMULATION BY MODULATING INTESTINAL FLORA HOMEOSTASIS} Marine Drugs of Ministry of Education, Shandong Provincial Key Laboratory of Glycoscience and Glycotechnology, School of Medicine and Pharmacy, Ocean University of China, Qingdao, 266003, China; Laboratory for Marine Drugs and Bioproducts, Pilot National Laboratory for Marine Science and Technology (Qingdao), Qingdao, 266237, China; Institute of Precision Medicine, The First Affiliated Hospital of Sun Yat-Sen University, Sun Yat-Sen University, Guangzhou, 510080, China; ${ }^{2}$ Key Laboratory of Marine Drugs of Ministry of Education, Shandong Provincial Key Laboratory of Glycoscience and Glycotechnology, School of Medicine and Pharmacy, Ocean University of China, Qingdao, 266003, China; Laboratory for Marine Drugs and Bioproducts, Pilot National Laboratory for Marine Science and Technology (Qingdao), Qingdao, 266237, China

10.1136/gutjnl-2021-IDDF.55

Background Polysaccharides from marine red algae possess a variety of biological activities. And, our previous study illustrated that the agaropectin-derived oligosaccharides from Gloiopeltis furcata (SAOs) activated AMPK signaling pathway in vitro. However, the effects of SAOs on alleviating lipid accumulation in vivo and its underlying mechanism are not clear.

Methods C57BL/6J mice were randomly divided into five groups of ten mice each. Control group: mice were fed a normal chow diet. And, the other four groups were continuously fed an HFD for 24 weeks to establish the obesity model. After 24 weeks, the Metf, SAOs-L, and SAOs-H groups received either metformin $(225 \mathrm{mg} / \mathrm{kg} / \mathrm{d}$ dissolved in saline) or SAOs $(100$ or $300 \mathrm{mg} / \mathrm{kg} / \mathrm{d}$ dissolved in saline), while the Control and Model groups were given aliquots of saline. Saline and the drugs were given by oral gavage for the final six weeks of the experimental period.

Results We found that SAOs decreased the body weight (about 3 grams) and the adiposity index (from 7 to 5) after a 6 week treatment. In addition, SAOs alleviated lipid accumulation in the liver, perirenal fat, and epididymal fat tissues. Investigation of the underlying mechanism showed that the cecal microbiota dysbiosis in HFD-fed mice were ameliorated after SAOs treatment, including significantly increasing the relative abundance of Alistipes, while reducing the relative abundance of Helicobacter. In addition, Spearman's correlation analysis indicated that changes in the cecal microbiota could regulate lipid accumulation, oxidative stress, inflammation.

Conclusions The present study confirmed that SAOs could effectively alleviate HFD-induced fat accumulation, partly through regulating cecal microbiota by fostering the preferential growth of probiotics and suppressing the relative abundance of harmful bacteria. In summary, our study illustrated that SAOs could be further developed as a potential pharmaceutical agent for obesity.

\section{IDDF2021-ABS-0198 CANAGLIFLOZIN ALLEVIATES DIABETIC CARDIOVASCULAR DISEASE VIA LIPID LOWERING, MITOCHONDRIAL HOMEOSTASIS, AND GUT MICROBIOTA REGULATION}

\begin{abstract}
${ }^{1,2,3}$ Xueliang Wang* ${ }^{1,2}$ Zhe Wang, ${ }^{1,2}$ Di Liu, ${ }^{1,2}$ Hao Jiang, ${ }^{1,2}$ Chao Cai, ${ }^{1,2}$ Guoyun Li, ${ }^{1,2}$ Guangli Yu. ${ }^{1}$ Key Laboratory of Marine Drugs of Ministry of Education, Shandong Provincial Key Laboratory of Glycoscience and Glycotechnology, School of Medicine and Pharmacy, Ocean University of China, Qingdao, China; ${ }^{2}$ Laboratory for Marine Drugs and Bioproducts, Pilot National Laboratory for Marine Science and Technology (Qingdao), Qingdao, China; ${ }^{3}$ Institute of Precision Medicine, The First Affiliated Hospital of Sun Yat-Sen University, Sun Yat-Sen University, Guangzhou, China

\subsection{6/gutjnl-2021-IDDF.56}

Background T2DM is associated with CVD. SGLT2i represent the most effective class of oral medications for the treatment of T2DM, and have been shown to improve generalized vascular dysfunction.

Methods T2DM was induced by HFD to male C57BL/6J mice for 24 weeks. After that, HFD-fed mice were treated with Cana $(50 \mathrm{mg} / \mathrm{kg} / \mathrm{d})$ by oral gavage for 6 weeks. Myocardial mitochondrial morphology and cardiovascular abnormalities were assessed by TEM, immunofluorescence staining and Masson's staining; lipids profile and oxidative stress state were assessed by corresponding biochemical assay kits and immunohistochemical staining; the myocardial injury was characterized by H\&E staining, TUNEL, and ELISA assays; colonic gut microbiota was assessed by $16 \mathrm{~S}$ rDNA sequencing. 